\title{
Projection-Neurons That Send Axons Through the Lumbar Spinal Cord of the Chick Embryo Are Not Obviously Distributed in a Segmentally Repetitive Pattern
}

\author{
G. Schlosser and K.W. Tosney \\ Biology Department, The University of Michigan, Ann Arbor
}

Earlier studies have shown that the formation of the segmentally repetitive pattern of spinal nerves in avian embryos depends upon the segmental arrangement of the somites and does not reflect an intrinsic pattern of the spinal cord. These studies could not, however, rule out the possibility that some elements of the central nervous system are intrinsically segmented. The question remained, for instance, whether projection-neurons are distributed in a segmentally repetitive pattern within the spinal cord. To address this question, horseradish peroxidase was injected into one or two segments of the lumbar spinal cord of chick embryos, thereby labeling projection-neurons that had ascending or descending axons passing through the injection site. In all stages examined, the number of labeled projection-neurons in the anterior and posterior halves of each segment did not differ significantly. In addition, segmentally repetitive peaks or troughs in the numbers of labeled projection-neurons were not detected in the center of each segment. Three subpopulations of projection-neurons, defined by their position along the dorsal-ventral axis of the spinal cord, were also not segmentally distributed by these criteria. While these results do not rule out the possibility that subpopulations of projection-neurons defined by dendritic morphology, functional class, or some other parameter are segmentally arranged or that there is a basic modular repetition of neural populations from segment to segment, they do show that projectionneurons as a class and subpopulations of these neurons defined by their dorsal-ventral position are not obviously distributed in a repetitive segmental pattern.

Key words: segmentation, metamerism, neural development, pattern formation, long projection neurons

\section{INTRODUCTION}

The concept that the central nervous system may be organized on a basic segmental plan is long standing and is of interest both to developmental neurobiologists and to those who study evolution. Evidence that neurons and targets may have a segmental identity and that this may help to mediate the development of specific innervation patterns [cf., Goodrich, 1906; Wigston and Sanes, 1982; Moody and Jacobson, 1983], while it may not explain innervation of limb muscles [Keynes et al., 1987; Lance-Jones, 1988; Tosney, 1988a], brings new enthusiasm to the search for intrinsic segmental differences in the nervous system. However, the degree to which particular elements of the nervous system are arranged in a segmental pattern, whether a particular spatial pattern within the nervous system is intrinsic or imposed by other tissues, and the functional importance of neural segmentation remain in most cases unclear.

Large-scale features of the nervous system that are segmentally arranged have been most studied and in these cases the pattern appears extrinsically derived. For instance, the spinal nerves and the trunk ganglia derive their segmental pattern through direct interactions with the adjacent somites [Lehmann, 1927; Detwiler, 1934; Weston, 1963; Keynes and Stern, 1984; reviewed by Tosney, 1988b]. The segmental arrangement of the neuromeres (bulges of the neural tube), long thought to reflect an intrinsic segmental pattern, may simply reflect the physical relations between the neural tube and the supporting cranial somitomeres [Meier and Tam, 1982] or somites [see Neal, 1918], particularly since the neuromeric bulges of the spinal cord arise in exact temporal coincidence with flanking somites and disappear as somites disintegrate [Vaage, 1969]. There is, in addition, no segmental pattern of mitotic activity [Hamburger, 1948; Tucket et al., 1985] that could support a proliferation model like that proposed by Bergquist and Källén [1954] for the intrinsic production of neuromeres.

Mr. Schlosser is now at Pechsteinstrasse 5, D-8500 Nuremberg 60, Federal Republic of Germany.

Received May 4, 1988; revised June 7, 1988; accepted June 10, 1988.

Address reprint requests to Dr. Kathryn W. Tosney, Natural Science Building, University of Michigan, Ann Arbor, MI 48109. 
One essential question that remains to be resolved is whether morphological or functional groups of central neurons are arranged in a segmental pattern and, if so, whether this pattern is intrinsic or imposed by other tissues. A segmentally repetitive arrangement of individually recognizable types of neurons has been described for Amphioxus [Bone, 1960] and a variety of "lower" vertebrates, ranging from the lamprey [Whiting, 1948] to zebrafish [Metcalfe et al., 1986; Eisen et al., 1986; Westerfield et al., 1986] and Xenopus [Nordlander, 1984]. However, there is much less evidence for a segmental organization of populations within the CNS of "higher" vertebrates. For instance, the nuclei of Kölliker or Hoffmann's nuclei that develop in the ventrolateral white column of the spinal cord of reptiles and birds are actually a continuous column of neurons with thickenings (more numerous cells) between the ventral nerve roots [Kölliker, 1902; Huber, 1936]. Since these nuclei form after the ventral roots, their apparent segmental pattern may be merely a consequence of the segmental position of axon outgrowth. The "microsegmentation" of alternating ipsi- and contra-lateral projecting neurons that has been described for the rat spinal cord [Altman and Bayer, 1984] is a regular and intrinsic repetitive pattern but the repetitive units are much smaller than embryonic segments. The preganglionic sympathetic neurons are grouped in irregularly spaced clusters that do not correspond to segments [Morgan et al., 1986] and motoneurons that innervate the limbs show no spatial [Landmesser, 1978] or temporal [Lim and Keynes, 1986] segmental pattern. However, motoneuron pools innervating muscles that are confined to a single somitic segment may be serially repeated in successive segments [see Hollyday and Hamburger, 1977; Tosney, 1988b]. If so, then thoracic segments in which motoneurons primarily innervate metamerically arranged muscles could comprise some sort of "modular units" along a segmental plan. However, we do not have enough information about the distribution of other populations along the longitudinal axis of the spinal cord to begin to realistically assess this possibility.

The present study addressed the issue of segmental patterning in the central nervous system by asking whether projection-neurons (neurons with long ascending or descending axons) are segmentally organized within the spinal cord of the chick embryo. We examined projection-neurons that had extended axons through lumbar spinal cord regions, since these would include somata in thoracic regions where there may be a basic metameric organization, as well as in lumbosacral regions where motoneuron pools are not arranged segmentally. We found no segmentally repetitive pattern in the numbers of projection-neurons when these are considered as a class or when they are divided into subpopulations defined by their dorsal-ventral position within the cord from the stages when these neurons first extend axons through the stages when motor innervation is established.

\section{MATERIALS AND METHODS}

Chick (Gallus domesticus) embryos of stages 17 to 32 [Hamburger and Hamilton, 1951] were decapitated and eviscerated in an oxygenated bath of Tyrode's solution at room temperature and the cartilage covering the ventral spinal cord was removed. A $10 \%$ solution of horseradish peroxidase (HRP) in Tyrode's solution was pressure injected into one or two lumbar segments of the spinal cord (see Table I). Following incubation for five

\section{TABLE I. Embryos Used in This Study ${ }^{*}$}

\begin{tabular}{|c|c|c|c|}
\hline $\begin{array}{l}\text { Embryo } \\
\text { number }\end{array}$ & Stage & $\begin{array}{c}\text { Injection } \\
\text { site }\end{array}$ & $\mathbf{N}$ \\
\hline GS 54 & 17 & LS $31(?)$ & - \\
\hline GS 55 & 17 & LS 31 (?) & - \\
\hline GS 52 & 18 & LS 31 (?) & - \\
\hline GS 53 & 19 & LS 31 (?) & - \\
\hline OR 114 & 20 & alt. hemisegm. & 8 \\
\hline GS 39 & 21 & LS 31 & 12 \\
\hline GS 40 & 21 & LS 31 & 1 \\
\hline OR 112 & 21 & alt. hemisegm. & 4 \\
\hline OR 113 & 21 & alt. hemisegm. & 3 \\
\hline OR 118 & 21 & alt. hemisegm. & 3 \\
\hline GS 35 & 23 & LS 31 & 4 \\
\hline OR 111 & 23 & LS 1 l, LS 3 r & 7 \\
\hline OR 107 & $23+$ & LS 11, LS $3 r$ & 7 \\
\hline GS 8 & 24 & LS 3 l, LS 3 r & 3 \\
\hline GS 37 & 24 & LS 31 & 12 \\
\hline OR 108 & 24 & LS 11, LS 3 r & 5 \\
\hline OR 84 & $24+$ & LS 11 , LS 3 r & 7 \\
\hline OR 106 & $24+$ & LS 1 I, LS 3 r & 10 \\
\hline OR 109 & $24+$ & LS 1 l, LS 3 r & 9 \\
\hline GS 34 & 25 & LS 31 & 7 \\
\hline OR 104 & 25 & LS 11, LS 3 r & 8 \\
\hline OR 110 & 25 & $\operatorname{LS} 11, \operatorname{LS} 3 \mathrm{r}$ & 8 \\
\hline OR 116 & $25+$ & LS $11, \mathrm{LS} 3 \mathrm{r}$ & 8 \\
\hline OR 100 & 26 & LS $11, \mathrm{LS} 3 \mathrm{r}$ & 14 \\
\hline GS 19 & 26 & LS 31 & 7 \\
\hline GS 10 & 27 & LS 31 & 17 \\
\hline GS 20 & 27 & LS 31 & 7 \\
\hline GS 21 & 27 & LS 31 & 18 \\
\hline GS 42 & 27 & LS 31 & 10 \\
\hline GS 43 & 27 & LS 31 & 4 \\
\hline GS 49 & 30 & LS 31 & 10 \\
\hline GS 50 & 30 & LS 31 & 12 \\
\hline GS 28 & 32 & LS 31 & 17 \\
\hline
\end{tabular}

*Summary of the embryos used in this study. $\mathrm{N}$ : number of hemisegments per embryo included in the analysis; LS 3: lumbosacral segment 3; LS 1: lumbosacral segment 1; 1 : left; $r$ : right; alt. hemisegm: injections were made in alternating left and right halves of successive segments. The axial level of injection sites for stages 17 to 19 could not be determined with certainty, but was approximately at level LS 3. 
$\mathrm{hr}$ in oxygenated Tyrode's solution at 31 to $34^{\circ} \mathrm{C}$, the embryos were fixed overnight in $2.5 \%$ glutaraldehyde in $0.1 \mathrm{M}$ cacodylate buffer, rinsed for 7 days in TRIS buffer, and then processed with diaminobenzidine (DAB) for visualization of HRP-containing cells. After rinsing briefly in $0.2 \mathrm{M}$ cacodylate buffer and postfixing overnight in $2 \%$ osmium tetroxide in $0.1 \mathrm{M}$ cacodylate buffer, the embryos were dehydrated in ethanol and propylene oxide and embedded in Epon-Araldite. The blocks were polymerized at $60^{\circ} \mathrm{C}$. Each embryo was serially sectioned at 10 to $25 \mu \mathrm{m}$ (usually $15 \mu \mathrm{m}$ ) transverse to its long axis on a Leitz rotary microtome. Each series of sections extended from the tail up to the lower brachial level of the embryo. For details of this procedure see Tosney and Landmesser [1986].

The segments of the embryo are initially obvious and can be defined by the distribution of the somites; however, this criterion is inadequate later in development when the transition between somites becomes less distinct [see Tosney, 1988b]. Therefore, we defined the anterior segment boundaries as beginning in the most anterior section where the spinal nerve traversed somitic tissue (Fig. 1B). This criterion is valid since spinal nerves form only in the anterior portion of each somite [see Keynes and Stern, 1984] and, after stage 19, ventral roots are seen only in the anterior of each segment [Tosney, 1988b]. In addition, a criterion for segmental organization dependent on a neural feature is preferable because it minimizes complications due to developmental alterations in somitic tissue that could theoretically shift the period or phase of the neural and mesodermal segmental systems. The boundaries of the left and right halves of each spinal cord segment were assessed separately to minimize the distorting effects of oblique sections. The distribution of labeled projection-neurons and morphology of the spinal cord was reconstructed at each stage by making camera lucida tracings of serial sections (see Fig. 2A-F).

Since HRP injected into neural tissue is taken up by axons at the injection site and distributed throughout the entire neuron [Tosney and Landmesser, 1986], our injections labeled the cell bodies of projection-neurons that had sent ascending or descending axons through the injection site. The somata of projection-neurons were successfully labeled in 33 out of 55 embryos. The total number of labeled cells per segment varied for two reasons. First, the number of axons that take up the label at the injection site appears to be random: some injections labeled more projection-neurons than others and the population of projection-neurons that becomes labeled seems to be a random sample of the total population present [see Tosney and Landmesser, 1986]. Second, the number of labeled projection-neurons decreased with distance from the injection site, presumably because fewer neurons with long projections are present but also because the axonal transport of HRP to distant neurons may be limited.
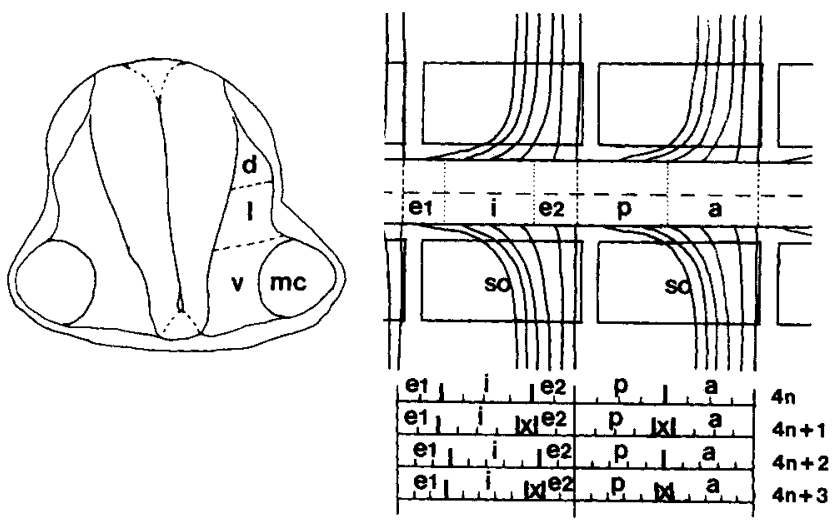

$1 \mathrm{~A}$

$1 B$

Fig. 1. A: Subpopulations of projection-neurons were categorized as dorsal (d), lateral (l), or ventral (v) according to their position in the mantle zone, as illustrated in this camera lucida tracing of a cross section through the lumbar level of the spinal cord of a stage 27 chick embryo. mc, motor column. B: Determination of segment boundaries and subdivision of segments. At top, two spinal cord segments with flanking somites (so) are schematized. The anterior segment boundary (dashed ventral line) was defined as beginning in the most anterior section where the spinal nerve (pictured as six lines) traversed somitic tissue and all the sections between two segment boundaries were regarded as a single segment. Segments were subdivided in two ways. To look for peaks or troughs in the number of projection-neurons in the center of each segment, each hemisegment (left or right side of a segment) was subdivided into exterior $(\mathrm{e}=\mathrm{e} 1+\mathrm{e} 2)$ and interior (i) portions of equal size (left). To look for peaks or troughs in the number of projection neurons in the anterior of each segment, each hemisegment was subdivided into an anterior (a) and posterior (p) portion of equal size (right). The diagram at bottom illustrates serial sections through each segment. To assure that each subdivision was of an equal size, one section had to be discarded (indicated by $X$ ) whenever an odd number of sections $(4+1$ or $4 n+3$, where $n=1-5$ ) constituted one segment. The exact mode of subdivision depended on the number of sections and is illustrated for 8 (generally, $4 n), 9(4 n+1), 10$ $(4+2)$, and $11(4 n+3)$ sections per segment.

We looked for two possible segmentally repetitive patterns of distribution by subdividing each segment into parts as shown in Figure 1B. Right and left halves (hemisegments) of the spinal cord were treated separately. First, to determine whether projection-neurons were preferentially located in the anterior or posterior part of each segment, each hemisegment was divided into an anterior and posterior portion of equal size and the number of labeled neurons in each half was counted. Second, to determine whether the number of these neurons increased or decreased in the center of each segment, each hemisegment was divided into an exterior and interior 


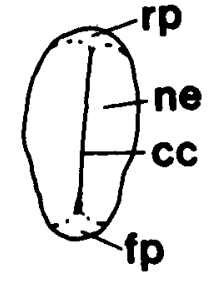

st 17

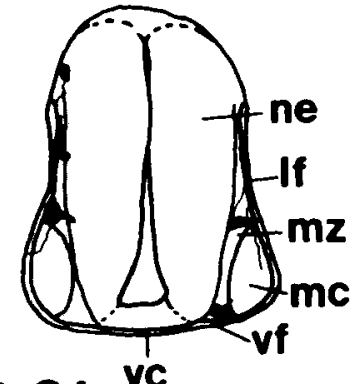

st 21

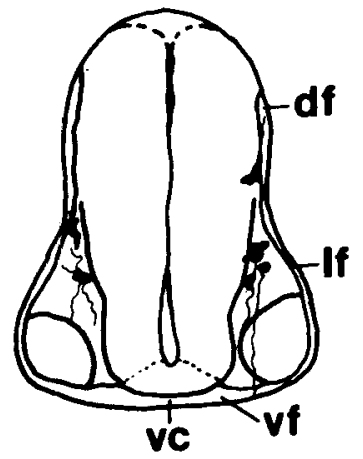

st 24
$100 \mu \mathrm{m}$
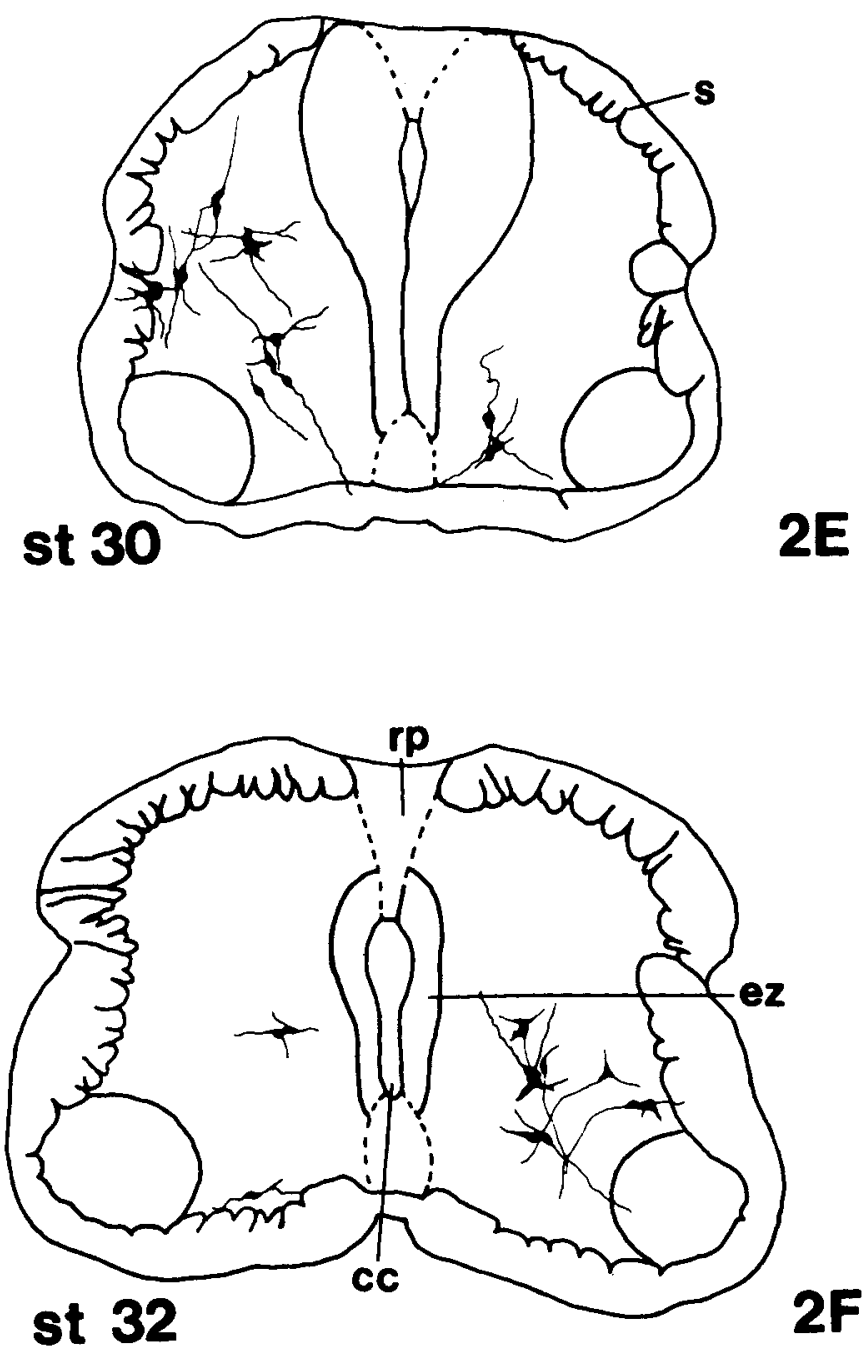

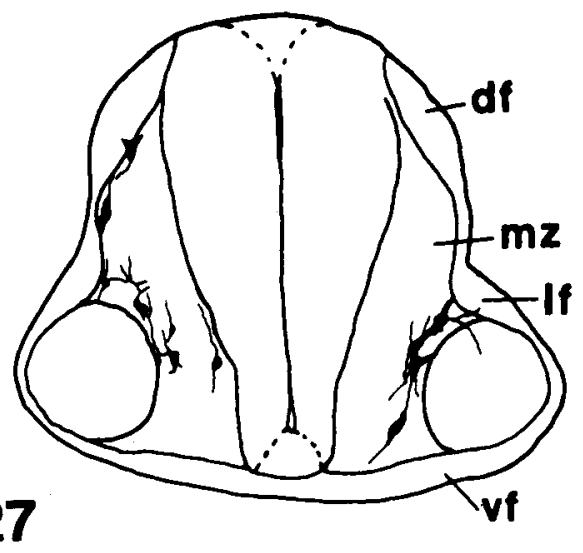

2D
Fig. 2. Overview of the early development of the spinal cord. Dorsal is toward the top. All figures are composites of camera lucida tracings of cross sections through the spinal cord at lumbar levels 2 and 3. By stage 17 (A) the spinal cord is still an undifferentiated tube of mitotically active neuroepithelium (ne); roof plate (rp) and floor plate (fp) can be distinguished. The central canal (cc) is a long dorsoventral slit. At stage 21
(B) the motor column (mc) is distinct and the first detectable projection-neurons constitute the mantle zone $(\mathrm{mz})$ lateral to the neuroepithelium. Projection-neurons are predominantly located dorsolateral to the motor column at this stage and send axons up and down the cord, establishing the ventral and lateral funiculi (vf and lf); few projection-neurons cross the (Fig. 2 legend continues on next page) 
portion of equal size and the number of labeled neurons in each half was counted. To assure an equal size of the subdivisions in each case, one section was discarded whenever an odd number of sections constituted one segment. Hemisegments at or adjacent to the injection site that obviously contained label in extracellular spaces were excluded from the analysis because neurons other than projection-neurons were also labeled. All labeled somata of projection-neurons were counted in each subdivision of the spinal cord along the longitudinal axis and these were also categorized by their dorsal-ventral position as shown in Figure 1A.

To obtain numbers that could be compared between different hemisegments, the data were standardized and expressed as a proportion: the number of labeled projection-neurons in each of the subdivisions was divided by the total number of labeled projection-neurons in the respective hemisegment. These proportions are slightly distorted by discarding one section in each segment with an odd number of sections but this tends to exaggerate rather than diminish possible differences between the subdivisions. The Wilcoxon signed rank test was used to determine if there were statistically significant $(P<0.05)$ differences in the proportions of labeled cells in anterior versus posterior or interior versus exterior parts of the hemisegments. Each embryo with more than eight useful hemisegments was first analyzed individually in this manner.

The neurons were examined at several stages, beginning shortly after axonogenesis, to reveal any early segmental pattern that might be obscured later in development. To determine if a stage-dependent pattern was present, all hemisegments were pooled by embryonic stage and then analyzed as described above. For those not familiar with Hamburger and Hamilton [1951] staging criteria, the approximate correspondence between embryonic stages and days of incubation is as follows: stage 17, 21/2 days; stage 19, 3 days; stage $21,3 \frac{1}{2}$ days; stage 24,4 days; stage 27,5 days; stage 29,6 days; and stage 31,7 days. The embryo hatches at 21 days.

\section{(Fig. 2 legend continued)}

ventral commissure $(\mathrm{vc})$ to the contralateral side. The dorsal funiculus (df) appears at stage 24 (C). By stage 27 (D) the mantle zone has broadened, especially in the alar plate (dorsal) and the white matter ( $\mathrm{df}$, If, and vf) has become more prominent. The projection-neurons are predominantly oriented along a line from the dorsolateral to ventromedial aspect of the spinal cord. Further broadening of the alar plate at stage 30 (E) gives the spinal cord a more rectangular outline. The dendritic trees of the projection-neurons are more widely branched and the grey matter exhibits little "spines" (s) where it borders the white matter. Beginning at stage 30 the mitotic activity in the neuroepithelium decreases and at stage 32 (F) the neuroepithelium assumes the appearance of an ependymal zone lining the reduced central canal and the roof plate has become more elongated.

\section{RESULTS}

\section{Overview of the Early Development of the Spinal Cord}

The development of projection-neurons during the stages examined in this study is first briefly described and integrated with previous information to provide a framework for the analysis of their distribution pattern. At stage 17 (Fig. 2A), the spinal cord is still an undifferentiated neuroepithelium and HRP injected into the spinal cord labels only neuroepithelial cells. During the period of initial motor axon outgrowth, the first projectionneurons ["arcuate cells," Hamburger, 1948; "circumferential cells," Holley, 1982] are born in the spinal cord (stage 19), giving rise to the "mantle zone" lateral to the dividing neuroepithelium [Ramon y Cajal, 1890; Hamburger, 1948; Holley, 1982]. We found that these first projection-neurons are preferentially located dorsolateral to the emerging motor column (Fig. 2B), confirming the similar report of Shneiderman et al. [1986]. Axons ascend or descend from the somata for about one segment in the spinal cord by stage 20 and establish the ventral and lateral funiculus of the white matter. Some projection-neurons project through the ventral commissure as early as stage 20 [much earlier than reported by Holley, 1982] and ascend or descend on the contralateral side but these contralateral projections do not become prominent until stage 27 (see Figs. 3-6).

By stage 24 (Fig. 2C) the dorsal funiculus of the white matter is visible and ascending fibers from the lumbosacral region have reached the brachial level [see

Figs. 3-6. The number of projection neurons in each subdivision of a segment in four representative embryos at different stages of development are shown. In each figure, $\mathbf{A}$ shows the number of labeled projection-neurons in the posterior (black columns) and anterior (grey columns) subdivisions of each segment and $\mathbf{B}$ shows the number of labeled projection-neurons in the exterior (black column) and interior (grey column) subdivisions of each segment. Positive counts represent the left and negative counts the right side of the cord. The left hemisegments indicated by an $X$ in each figure contained the injection site and were discarded.

Fig. 3. Stage-21 embryo (GS 39 in Table I); each graph extends from lumbosacral (LS) segment 4 (at left) to thoracic (T) segment 4 (at right).

Fig. 4. Stage-24 embryo (GS 37); each graph extends from LS6 (at left) to T7 (at right).

Fig. 5. Stage-27 embryo (GS 10); each graph extends from LS6 (at left) to T5 (at right).

Fig. 6. Stage-30 embryo (GS 50); each graph extends from LS6 (at left) to T6 (at right). 

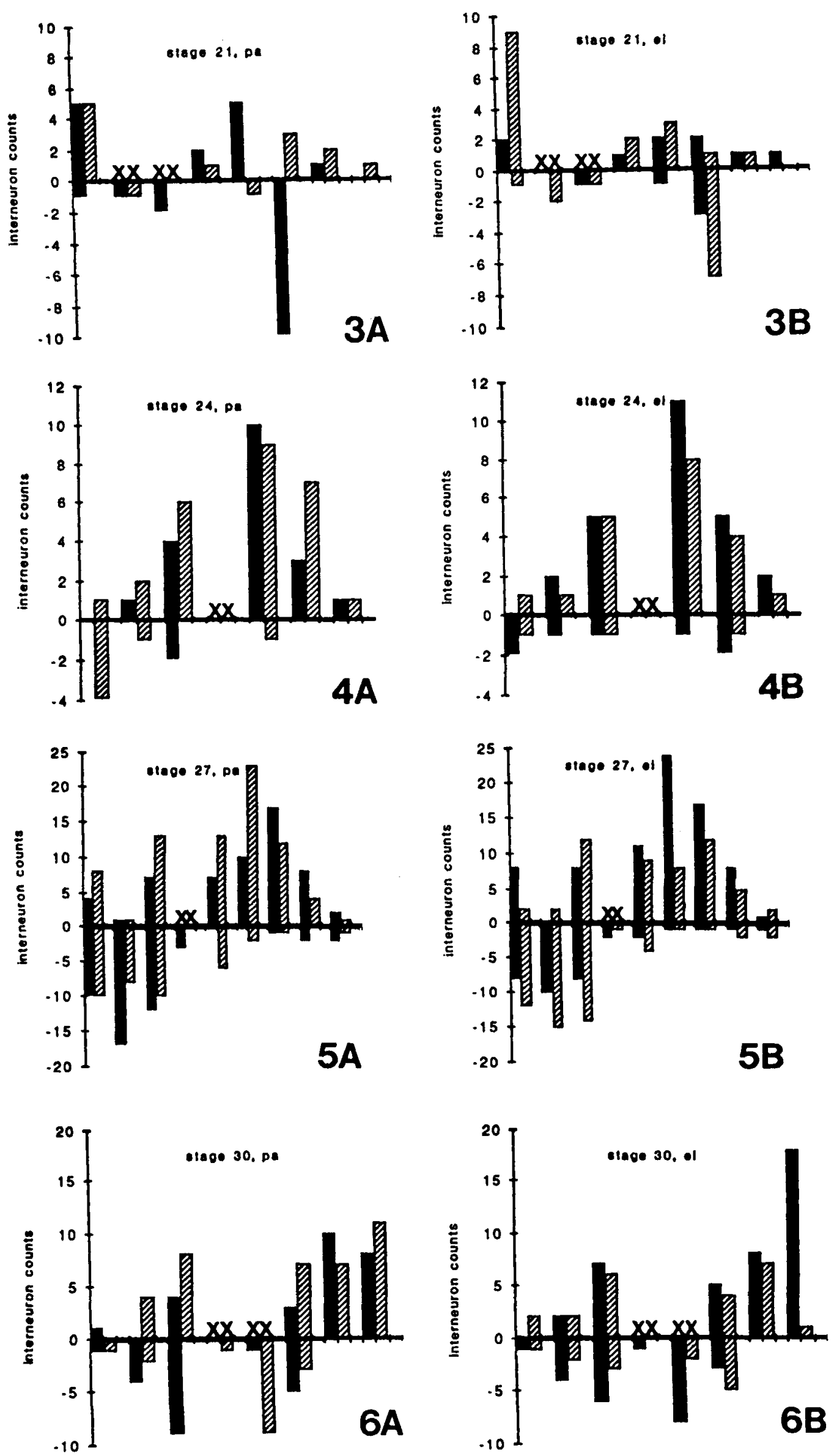
also Shneiderman et al., 1986] and can mediate propriospinal integration of movements between fore and hind limbs. The ascending interconnection of lumbosacral and brachial levels precedes their descending interconnection [see also Oppenheim, 1975] and is established prior to the closure of the intrasegmental reflex arc in stage 31 [Windle and Orr, 1934; Nornes et al., 1980a]. The development of the basal plate precedes the development of the alar plate of the spinal cord (Fig. 2B-E), reflecting the different temporal pattern of mitoses ventrally and dorsally: the peak of mitotic activity in the basal plate (ventral) is at stage 20 , whereas the mitotic activity of the alar plate (dorsal) reaches its maximum as late as stage 29 [Hamburger, 1948].

By stage 27 (Fig. 2D) the mantle zone has increased enormously in size and has become prominent in the alar plate as well. Labeled projection-neurons are predominantly oriented along a line from the dorsolateral to ventromedial aspect of the spinal cord and many send axons through the ventral commissure to the contralateral side. According to earlier studies [Windle and Orr, 1934; Nornes et al., 1980b] the ventral and lateral white columns harbor mainly contralateral ascending fibers at this stage, whereas ipsilateral projections become predominant later. In the embryos examined in this study, however, ipsilateral projections were predominant at all stages analyzed (see Figs. 5 and 6).

By stage 30 (Fig. 2E) the grey matter of the alar plate has broadened [due to a peak of mitotic activity in the alar plate at stage 29; Hamburger, 1948], whereas the neuroepithelium has regressed further, especially in the basal plate. The predominant alignment of the projection-neurons along a dorsolateral to ventromedial line that was clearly visible in earlier stages is no longer obvious. Instead, projection-neurons appear to be oriented in various directions and their dendritic trees are more widely branched. This richer branching of the projection-neurons and an increased sprouting of unmyelinated collaterals from ascending and descending axons into the grey matter may lead to the formation of the "spines" of grey matter that we first see at stage 30 . Mitotic activity starts to decline in the alar plate at stage 30 , ending at stage 35 [Hamburger, 1948]. By stage 32 (Fig. 2F) the roof plate has increased in length and the neuroepithelium has become narrower and appears as an ependymal zone lining the reduced central canal.

\section{Distribution of Labeled Projection-Neurons}

The distribution of labeled projection-neurons along the longitudinal axis for four representative embryos of stages 21, 24, 27, and 30 is shown in Figures 3-6. The distribution is similar in the other embryos examined. Two histograms were plotted for each embryo: the first (A in Figs. 3-6) shows the distribution in the anterior versus posterior portions of each segment; the second (B in Figs. 3-6) shows the distribution in the exterior versus interior portions of each segment. With increasing distance from the injection site the number of projectionneurons that are labeled generally decreases. In some cases, there is another maximum of labeled projectionneurons two to four segments away from the injection site (e.g., Figs. 5 and 6) but this appears to be random since the position of the peaks is not consistent from embryo to embryo. Since there were no obvious differences between the ipsilateral and contralateral sides of the spinal cord in the segmental distribution of labeled projection-neurons, these were treated together.

Consistent differences in the number of labeled projection-neurons between anterior and posterior subdivisions on the one hand and between exterior and interior subdivisions on the other hand are not obvious in any of the four embryos shown in Figures 3-6. In particular, there is no obvious difference between thoracic and lumbosacral segments. A statistical analysis of all embryos examined with more than eight useful hemisegments reveals no statistically significant differences $(P<0.05)$ between the proportions of labeled cells in the anterior versus posterior or in exterior versus interior parts of the segments.

When hemisegments were grouped by stage, segmental differences were again not obvious. For instance, the means of anterior and of exterior proportions are close to 0.5 for each stage examined (Fig. 7) indicating that labeled projection-neurons are distributed equally in the anterior and posterior portions as well as in the exterior and interior portions of the hemisegments. There is no significant difference $(P<0.05)$ in their distribution in any of the embryonic stages with one exception: the differences between the exterior versus interior parts of hemisegments for stage 30 are insignificant only at $P<0.032$.

It is possible that different subpopulations of projection-neurons are segmentally distributed but have complementary patterns of distribution that cancel each other out when counts are pooled. To examine this possibility, three subpopulations of projection-neurons, defined by their position along the dorsoventral axis of the cord as in Figure 1A, were assessed separately. For each subpopulation the number of anterior or posterior, and exterior or interior divisions of hemisegments was counted in which labeled neurons were present. For example, ventral projection-neurons were found in the anterior division of 140 hemisegments and in the posterior division of 138 hemisegments (out of 242, see Table I). As shown in Figure 8A-C, there is no striking difference between the pattern of distribution of dorsal, lateral, and ventral classes of projection-neurons. The fluctuations are larger for the dorsal category (Fig. 8A) because fewer interneurons were labeled in the dorsal position in most of the embryonic stages. This underrepresentation 
may be due to the delayed development of the alar plate compared to the basal plate of the spinal cord or to the fact that the HRP was generally injected into the ventral spinal cord. None of these three categories showed preferential localization in anterior versus posterior or exterior versus interior parts of the hemisegments: the number of hemisegments with labeled projection-neurons in their anterior part is about the same as the number of hemisegments with labeled cells in their posterior part for each category. The same is true for exterior and interior parts of the hemisegments. There were also no obvious differences between the various stages (not shown). Thus, the results reported here give no indication that projectionneurons as a population or that subpopulations defined by their position along the dorsal-ventral axis have a segmentally repetitive pattern of distribution.

\section{DISCUSSION}

There are a number of segmentally repetitive patterns that can be envisioned in the central nervous system. One morphologically obvious pattern would be a periodic variation in the numbers of somata in a population. We looked for consistently higher or lower numbers of projection-neurons in the anterior portion or in the central portion of each segment and found no indication of a reiterative distribution from the time when projection neurons are first born (stage 19) past the time when motor axons have reached their targets (stage 27). The sole exception, the significant difference between external versus internal portions of segments at stage 30 , is unlikely to be important, since the difference is transitory. The absence of a segmentally repetitive pattern during early stages suggests that there is no pattern in the "birth" of projection-neurons, such that projection-neurons are born first in anterior parts of the segments and later in posterior parts or vice versa, as has been found for the zebrafish [see Myers et al., 1987]. If such a temporal pattern were present, we would expect to find a segmental distribution of projection-neurons in at least some of the stages examined before stage 30 , during the period when the majority of these neurons are born [see Hamburger, 1948] and we did not. This indicates that, if there is a segmental pattern of "birthdays" of projection-neurons, it is subtle or based on subclasses of these neurons. Moreover, since a segmental pattern was not detected at stages after motor axons have ramified within their limb targets [e.g., at stage 32; see Tosney and Landmesser, 1985a,b]. motor axons are unlikely to exert retrograde influences on the projection-neurons, rearranging them into a secondary segmental pattern.

These results demonstrate that there is no obvious repetitive pattern of segmentation with respect to the class of neurons that send long projection axons through the lurnbar spinal cord or with respect to subpopulations of these neurons that are defined by their dorsal-ventral position within the cord. Our study does not, however, rule out the possibility that there may be other subpopulations of projection-neurons, defined for example by the morphology of their dendritic trees or an identifiable position along the dorsoventral and mediolateral axes in the cord, that are distributed in a segmental fashion, or that there is some other repetitive pattern that is not coincident with segmental units defined by spinal nerve position, or that subpopulations may form modular units that are repeated in each segment. It is also important to
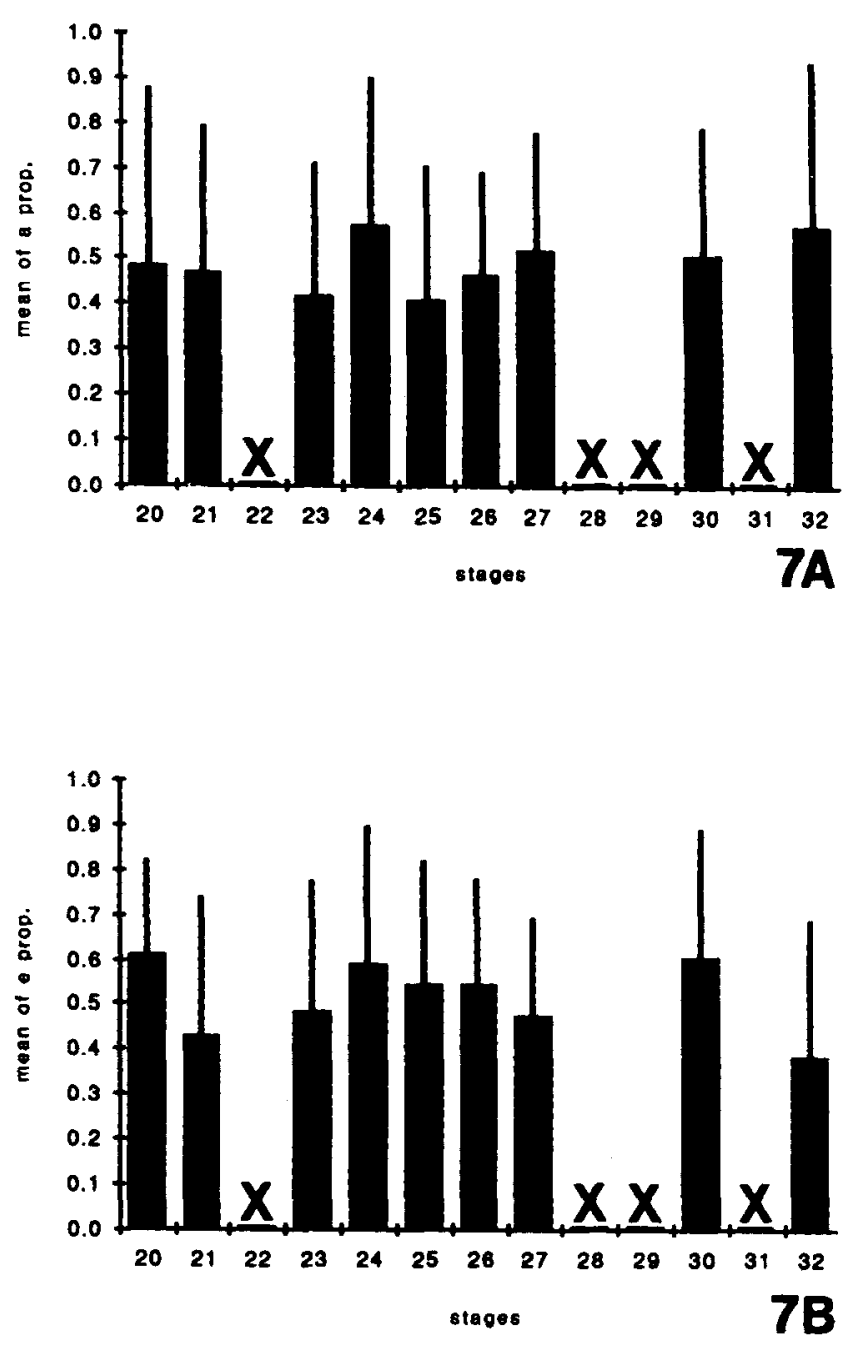

Fig. 7. The means of the proportions of (A) anterior projection-neurons per hemisegment and (B) exterior projectionneurons per hemisegment shown for each embryonic stage (indicated on the $\mathrm{x}$ axis). The positive standard deviations are indicated by vertical bars. These are large because values often fluctuated widely with $0 \%$ in some and $100 \%$ in other hemisegments. The number of hemisegments analyzed per stage is 8 (stage 20), 23 (stage 21), 18 (stage 23), 46 (stage 24), 31 (stage 25), 21 (stage 26), 56 (stage 27), 22 (stage 30), and 17 (stage 32). Data are not available for stages indicated by $\mathrm{X}$. 

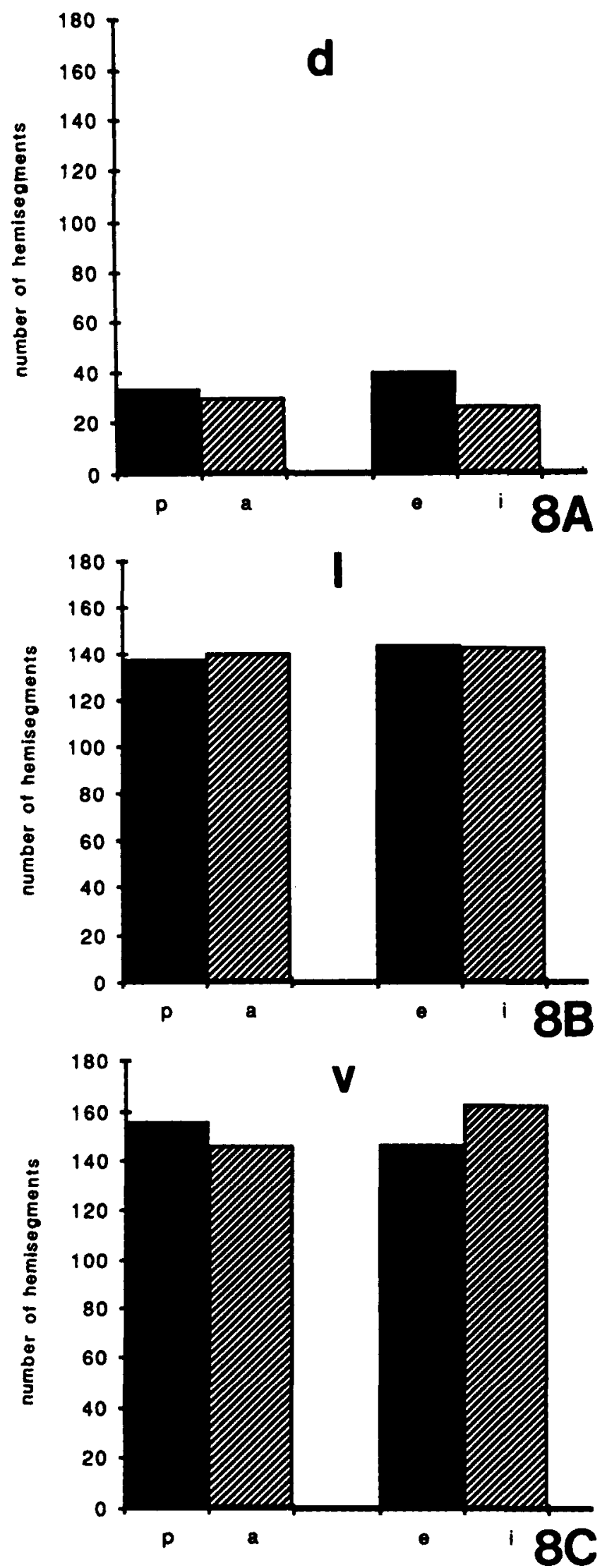

point out that a repetitive morphological pattern is not the only possible way in which segmentation could be expressed. For instance, some property (e.g., a molecular cell-surface label) could vary from one segment to the next in a discontinuous fashion, thereby specifying segment identity. If this were the case in the nervous system, neural somata would not necessarily be segmentally distributed along the longitudinal axis but neurons from different segments would be expected to have segmentspecific surface labels. Further studies are required to clarify whether there are segmental labels in the developing embryo and what, if any, role these play in the development of the vertebrate nervous system.

\section{ACKNOWLEDGMENTS}

We thank Sophia Tyrell and Sally Schroeter for technical assistance and Kate Barald, John Kuwada, and Sally Schroeter for reading early versions of the manuscript.

\section{REFERENCES}

Altman J, Bayer SA (1984): The development of the rat spinal cord. Berlin: Springer Verlag, $166 \mathrm{pp}$.

Bergquist H, Källén B (1954): Notes on the early histogenesis and morphogenesis of the central nervous system in vertebrates. $J$ Comp Neurol 100:627-659.

Bone Q (1960): The central nervous system in Amphioxus. J Comp Neurol 115:27-64.

Detwiler SR (1934): An experimental study of spinal nerve segmentation in Amblystoma with reference to the plurisegmental contribution to the brachial plexus. J Exp Zool 67:395-441.

Eisen IS, Myers PZ, Westerfield M (1986): Pathway selection by growth cones of identified motoneurons in live zebrafish embryos. Nature 320:269-271.

Goodrich ES (1906): Notes on the development, structure and origin of the median and paired fins of fish. Q J Microscop Sci 50:333-376.

Hamburger V. (1948): The mitotic patterns in the spinal cord of the chick embryo and their relation to histogenetic processes. J Comp Neurol 88:221-283.

Hamburger V, Hamilton HL (1951): A series of normal stages in the development of the chick embryo. J Morphol 88:49-82.

Holley JA (1982): Early development of the circumferential axonal pathways in mouse and chick spinal cord. J Comp Neurol 205:371-382.

Hollyday M, Hamburger V (1977): An autoradiographic study of the formation of lateral motor column in the chick embryo. Brain

Fig. 8. Numbers of hemisegments (out of a total of 242 examined) in which dorsal (d), lateral (l), and ventral (v) projection-neurons are present. A: The $\mathrm{x}$ axis indicates the type of segment subdivision: posterior (p) and anterior (a) or exterior (e) and interior (i). The number of hemisegments of each type that contained dorsal projection-neurons is shown as a column for each type of subdivision. B: Same as A for lateral projection-neurons. C: Same as A for ventral projection-neurons. 
Res 132:197-208.

Huber JF (1936): Nerve roots and nuclear groups in the spinal cord of the pigeon. J Comp Neurol 65:43-91.

Keynes RJ, Stern CD (1984): Segmentation in the vertebrate nervous system. Nature 310:786-789.

Keynes RJ, Stirling RV, Stern CD, Summerbell D (1987): The specificity of motor innervation of the chick wing does not depend upon the segmental origin of muscles. Development 99:565 575.

Kölliker A (1902): Weitere Beobachtungen über die HOFMANN'schen Kerne am Mark der Vögel. Anat Anz 21:81-84.

Lance-Jones C (1988): The effect of somite manipulation on the development of motoneuron projection patterns in the embryonic chick hindlimb. Dev Biol 126:394-414.

Landmesser L (1978): The distribution of motoneurones supplying chick hind limb muscles. J Physiol 284:371-389.

Lehmann FE (1927): Further studies on the morphogenetic role of the somites in the development of the nervous system of amphibians. I Exp Zool 49:93-131.

Lim TM, Keynes RJ (1986): Segmentation in the neural tube of the chick embryo. Soc Neurosci Abs 12:316.

Meier S, Tam PPL (1982): Metameric pattern development in the embryonic axis of the mouse. I. Differentiation of the cranial segments. Differentiation 21:95-108.

Metcalfe WK, Mendelson B, Kimmel CB (1986): Segmental homologies among reticulospinal neurons in the hindbrain of the zebrafish larva. J Comp Neurol 251:147-159.

Moody SA, Jacobson M (1983): Compartmental relationships between anuran primary spinal motoneurons and somitic muscle fibers that they first innervate. J Neurosci 3:1670-1682.

Morgan CW, DeGroat C, Nadelhaft I (1986): The spinal distribution of sympathetic preganglionics and visceral primary afferent neurons that send axons into the hypogastric nerves of the cat. J Comp Neurol 243:23-40.

Myers PZ, Eisen JS, Westerfield M (1986): Development and axonal outgrowth of identified motoneurons in the zebrafish. J Neurosci 6:2278-2289.

Neal HV (1918): Neuromeres and metameres. J Morphol 31:293315.

Nordlander RH (1984): Developing descending neurons in the caudal spinal cord of early Xenopus. J Comp Neurol 228:117-128.

Nornes HO, Hart H, Carry M (1980a): Pattern of development of ascending and descending fibers in embryonic spinal cord of chick. II. A correlation with behavioral studies. J Comp Neurol 192:133-141.

Nornes HO, Hart H, Carry M (1980b): Pattern of development of ascending and descending fibers in embryonic spinal cord of chick, I. Role of position information. J Comp Neurol 192:119-
132.

Oppenheim RW (1975): The role of supraspinal input in embryonic motility, a re-examination in the chick. J Comp Neurol 160:3750.

Ramon y Cajal S (1890): A quelle époque apparaissent les expansions des cellules nerveuses de la moëlle épinière du poulet? Anat Anz 5:609-613, 631-639.

Shneiderman A, Okado N, Oppenheim RW, Shiba R (1986): The development of propriospinal pathways in chick embryo spinal cord. Soc Neurosci Abs 12:1503.

Tosney KW (1988a): Proximal tissues and patterned neurite outgrowth at the lumbosacral level of the chick embryo: Partial and complete deletion of the somite. Dev Biol 127:266-286.

Tosney KW (1988b): Somites and axon guidance. Scanning Microsc 2:427-442.

Tosney KW, Landmesser LT (1985a): Development of the major pathways for neurite outgrowth in the chick hindlimb. Dev Biol 109:193-214.

Tosney KW, Landmesser LT (1985b): Specificity of early motoneuron growth cone outgrowth in the chick embryo. J Neurosci 5:2336-2344.

Tosney KW, Landmesser LT (1986): Neurites and growth cones in the chick embryo. Enhanced tissue preservation and visualization of HRP-labeled subpopulations in serial $25-\mu \mathrm{m}$ plastic sections cut on a rotary microtome. J Histochem Cytochem 34:953-957.

Tuckett F, Lim L, Morriss-Kay GM (1985): The ontogenesis of cranial neuromeres in the rat embryo I. A scanning electron microscope and kinetic study. J Embryol Exp Morphol 87:215228.

Vaage S (1969): The segmentation of the primitive neural tube in chick embryos (Gallus domesticus). Ergebn Anat Entw Gesch 41:1-88.

Westerfield M, McMurray JV, Eisen JS (1986): Identified motoneurons and their innervation of axial muscles in the zebrafish. $J$ Neurosci 6:2267-2277

Weston J (1963): A radioautographic analysis of the migration and localization of trunk neural crest cells in the chick. Dev Biol $6: 279-285$.

Whiting HP (1948): Nervous structure of the spinal cord of the young larval brook-lamprey. Q J Microscop Sci 89:359-384.

Wigston DJ, Sanes JR (1982): Selective reinnervation of adult mammalian muscle by axons from different segmental levels. Nature 299:464-467.

Windle WF, Orr DW (1934): The development of behavior in chick embryos; spinal cord structure correlated with early somatic motility. J Comp Neurol 60:287--307. 